\title{
INHIBITORY EFFECT OF ORGANOGERMANIUM COMPOUNDS ON THE BIOLOGICAL MAILLARD REACTION
}

\author{
Toshihio Osawa1, Shunro Kawakishi1, Mitsuo Akiba² and Norihiro Kakimoto² \\ ${ }^{1}$ Department of Applied Biological Sciences, Faculty of Agriculture, Nagoya University, Chikusa, \\ Nagoya 464-01, Japan \\ 2 Asai Germanium Research Institute, Izumihoncho, 1-6-4, Komae, Tokyo 201, Japan
}

\begin{abstract}
Glucose and amino-group of proteins form Schiff bases, whose reaction is reversible, and then Amadori products can be formed by rearrangement from the Schiff bases by the biological Maillard reaction. Many different types of advanced glycation end products (AGE) can be formed from Amadori products. Among AGE, formation of pentosidine must be more important biological and physiological marker of the Maillard reaction. Inhibitory effect of Ge-132 was observed on the formation of pentosidine by the reaction of arginine, ribose and lysine as well as the further progress reaction of Amadori product, fructose lysine, to pentosidine in the presence of arginine. These results indicated that organogermanium compounds, in particular Ge-132, inhibited both of formation of Amadori rearrangement adducts and further progress reaction to AGE, however, Ge132 was found to have no free radical scavenging activity. By the detailed ${ }^{1} \mathrm{H}-\mathrm{NMR}$ analyses of the reaction of Ge-132 with glucose or chemically synthesized glucofuranose derivatives, it was found that Ge-132 made an complex with glucofuranose form of glucose and inhibited the early stage of the Maillard reaction. Ge-132 was also found to make a complex with Amadori rearrangement adducts such as fructoselysine to stabilize and inhibit the futher progress of the Maillard reaction.
\end{abstract}

\section{INTRODUCTION}

Much attention has been focused on organogermanium compounds because of their physiological and biological activities. Synthesis of trialkylgermanium acetate was reported in 1962 by Kaars et al as the first biological active organogermanium compound, which demonstrated to have an antifungal activity ${ }^{1)}$. In 1968, Asai and Kakimoto reported the synthesis of organogermanium sesquioxide $(\mathrm{Ge}-132)^{2)}$. Many in vitro and in vivo experiments revealed that Ge-132 is a unique biological and physiological active compound of extremely low toxicity such as biological responce modifying (BRM) activity, antiviral activity, and regulation of calcium metabolism, alleviation of carcerous pain and osteoscope ${ }^{3)}$.

In 1912, Maillard found that glucose or other reducing sugars react with amino acids to form adducts that undergo a series of non-enzymatic browning reaction in the stored- or heattreated foods ${ }^{4}$ ). In recent years many reports have suggested that the Maillard reaction plays an important role of diseases associated with diabetes and aging. Maillard products have been observed in vivo in association with several long-lived proteins such as lens crystallines and collagen. The aldehyde group of glucose reacts with the amino groups of proteins to form Schiff base adducts, followed by Amadori rearrangement, and a series of dehydrations and rearrangements to form advanced glycation end products. (AGE) ${ }^{5}$ ).

Recently, Nakamura et al. reported that organogermanium compounds (2carboxyethylgermanium sesquioxide: Ge-132, and 2-Carboxy-2-aminoethylgermanium sesquioxide: Ge-385) were effective in suppressing the formation of AGE, which is suspected to contribute to the development of diabetic complication, from serum proteins, collagen and lens crystallins although the detailed mechanism is not clear 6). These backgrounds prompted us to investigate the chemical aspect of the mode of action of organogermanium compounds using the biomimic Maillard reaction and NMR technique. In this paper, inhibitory mechanism of Ge-132 and its related compounds on the Maillard reaction will be presented. 


\section{MATERIALS AND METHODS Chemicals}

Carboxyethylgermanium sesquioxide (Ge-132) was synthesized by the previously reported method ${ }^{3)}$. Acrylic acid was treated with trichlorogermane in conc. hydrochloric acid to form trichlorogermylpropanoic acid, followed by hydrolysis to form $\mathrm{Ge}-132$ together with minor product. Recently, the authors succeeded in introducing amino-group to Ge-132 in order to increase the solubildity in water. Ge-385 was synthesized by trichlorogermylation of $\alpha, \beta$-unsatulated amino acids and azulactones followed by hydrolysis 7$)$. For the comparison of antioxidative activity, Ge$014^{7)}$ and $\mathrm{Ge}-005^{8)}$ were also prepared by the previously reported method. Ge-014 is germanium sesquisulfide, which has the adamantane structure, and Ge-005 has structually related germanium sesquioxide structure. $N^{\alpha}$-tert-Boc- $N^{\varepsilon}$-fructose lysine $(F L)$ was synthesized by the previously reported method from $N^{\alpha}$-t-butoxycarbonyl-L-lysine $\left(N^{\alpha}\right.$-tert-Boc-Lysine) and glucose $\left.{ }^{9}\right)$. Authentic pentosidine was synthesized and purified by the reaction of ribose, Boc-arginine and Boc-lysine followed by the previously reported method 10 ).

3,5-Acetoneglucose was prepared by the reaction of glucose and acetone using $85 \%$ $\mathrm{H} 3 \mathrm{PO}_{4}$ and anhydrous $\mathrm{ZnCl} 2$ in the mechanically stirred suspension. D-Glucuronolactone and cyclohexane-1,2-diol were purchased from Aldrich Co. Ltd..

\section{Reaction conditions}

Formation of pentosidine was investigated by the reaction of ribose, Boc-arginine and Boclysine (10 mM, each) or $10 \mathrm{mM}$ of FL and Boc-arginine in $2 \mathrm{ml}$ of phosphate buffer $(\mathrm{pH} \mathrm{7.4,100}$ $\mathrm{mM}$ at $37^{\circ} \mathrm{C}$. Inhibitory effects of organogermanium compounds were examined at the concentration of $5 \mathrm{mM}$ and $10 \mathrm{mM}$. After reaction, $0.5 \mathrm{ml}$ of the reaction mixture was freeze-dried and dissolved in $0.5 \mathrm{ml}$ of trifluoroacetic acid to remove BOC by hydrolysis. After hydrolysis for 4 hours at room temperature, the reaction mixture was concentrated in vacuo, dissolved in $0.5 \mathrm{ml}$ $\mathrm{H}_{2} \mathrm{O}$ and $20 \mu \mathrm{l}$ of the solution was injected to HPLC for quantification of pentosidine. Separations were made on a $0.46 \times 25 \mathrm{~cm}$ Develosil ODS-5 (Nomura Chemicals Co. Ltd., Seto, Aichi) by application of a linear gradient of $3 \%-15 \%$ acetonitrile in $0.1 \%$ trifluoroacetic acid applied from 15 to $25 \mathrm{~min}$ after isocratic condition (3\% acetonitrile in $0.1 \%$ trifluoroacetic acid) for $15 \mathrm{~min}$.

\section{Instruments}

Separation of pentosidine was carried out on GULLIVER HPLC (JASCO Co. Ltd., Japan) with a model 821-FP fluorescence detector (JASCO Co. Ltd., Japan) at an excitation wave length of $335 \mathrm{~nm}$ and monitoring of emission at $385 \mathrm{~nm}$. NMR spectra were measured by $300 \mathrm{MHz} \mathrm{FT}$ NMR (Varian Co. Ltd., U.S.A.).

\section{RESULTS}

Inhibitory effects of organogermanium compounds on the formation of pentosidine In order to examine the detailed reaction mechanism, pentosidine was used as the

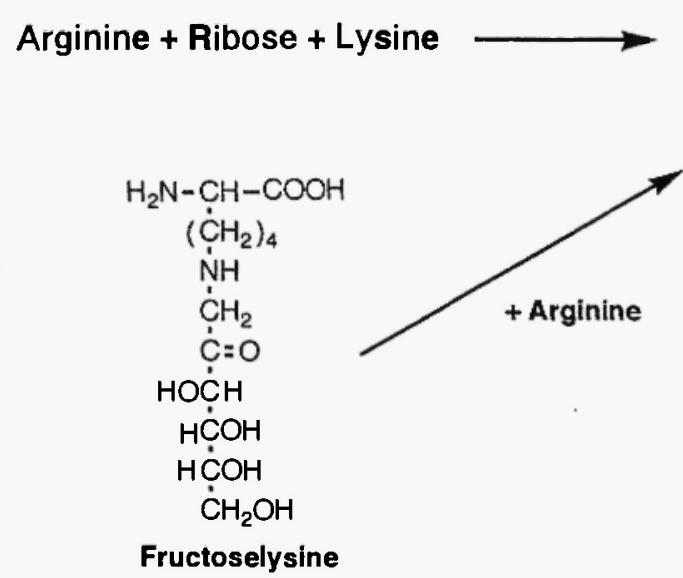<smiles></smiles>

Fig.1 Formation of pentosidine in biomimic reaction system 
biological marker for the progress of glycation. Pentosidine can be formed by the reaction of arginine, ribose and lysine, and also by the reaction of Amadori product (FL) with arginine (Fig. 1). At first, inhibitory effects of structually related organogermanium compounds on the formation of pentosidine from arginine, ribose and lysine were examined. As shown in Fig. 2, Ge-014, 005 and 132 were found to inhibit effectively although Ge-385 has less activity. Next, inhibitory effects of organogermanium compounds on the formation of pentosidine derived from Amadori compound (FL) and arginine were examined. This data (Fig. 3) showed exactly the same tendency as the inhibitory effect on the formation of pentosidine by the reaction of argnine, ribose and lysine.

Although Ge-385 was not so strong, other organogermanium compounds, Ge-014, 005 and 132, have also quite strong inhibitory effect.

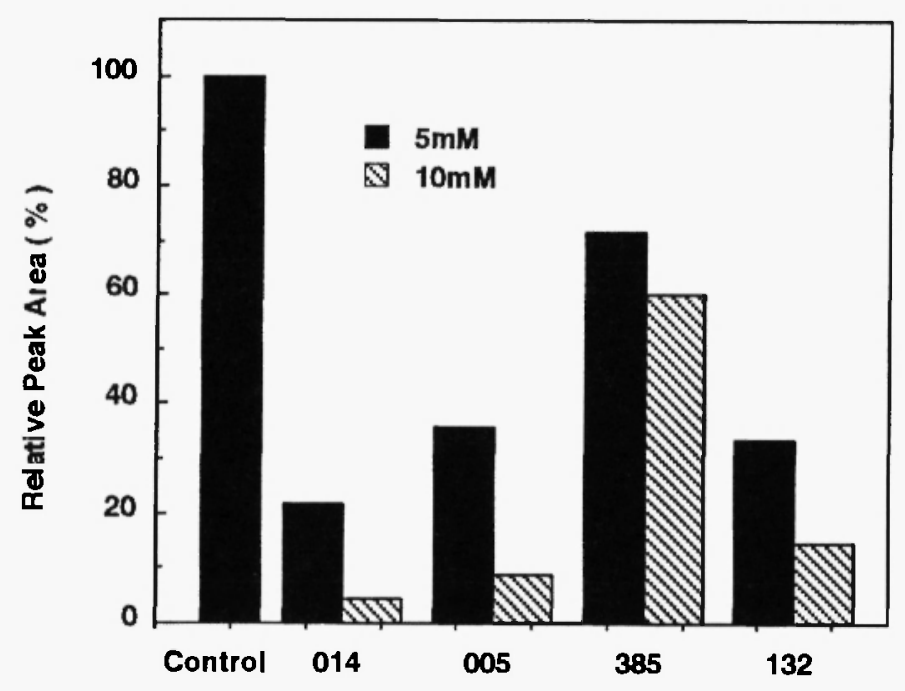

Fig.2 Inhibitory effects of organogermanium compounds on the formation of pentosidine derived from ribose, arginine and lysine

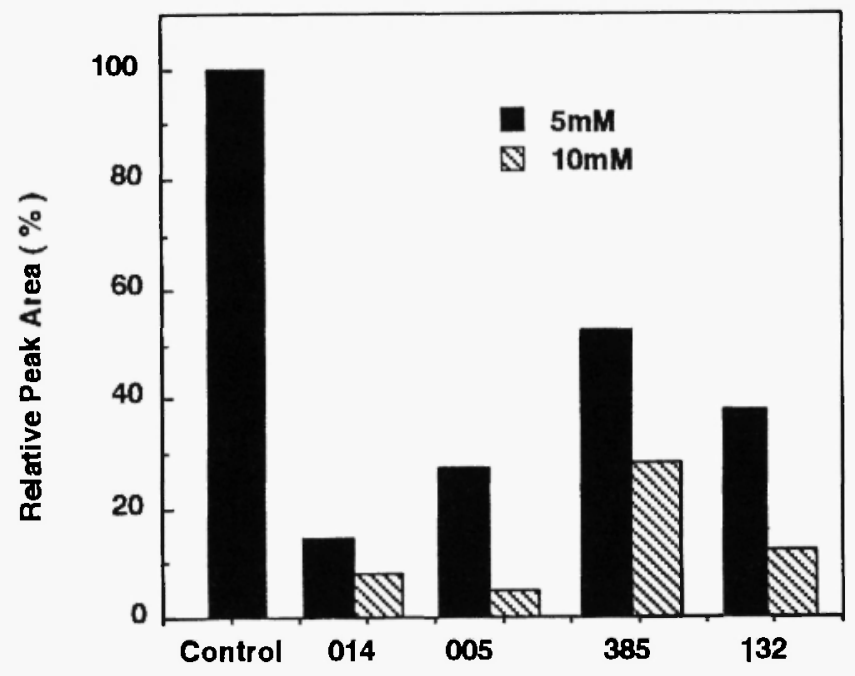

Fig.3 Inhibitory effects of organogermanium compounds on the formation of pentosidine derived from fructose lysine and arginine 


\section{Free radical scavenging activity of organogermanium compounds}

Recently, there are many indications that free radicals must play an important role for the biological Maillard reaction. It has been suggested that oxygen species such as hydrogen peroxide, superoxide anion radical and singlet oxygen agents attack polyunsaturated fatty acids in cell membranes, leading to lipid peroxidation. It was indicated that lipid peroxidation may lead to destabilization and disintegration of cell membranes, and cause many age-related diseases including development of diabetic complication. These background prompted us to investigate whether these organogermanium compounds may inhibit the lipid peroxidation or not.

Evaluation systems which we used are in vitro systems by rat liver microsome and rabbit erythrocyte ghost systems, and it was found that adamantane type germanium sesquisulfide, Ge014 only inhibited lipid peroxidation effectively, however, other types of organogermanium compounds had no antioxidative activity as shown in Table 1. Althogh the detailed antioxidative mechanism of Ge-014 was examined by the use of spin-trapping method and will be published elsewhere, Ge-014 were found to play an important role for inhibition of the lipid peroxidation promoted by the heme compounds associated with the phospholipids of erythrocyte and microsome membranes. These data suggested that organogermanium compounds do not act as the free radical scavengers of oxygen radicals formed during biological Maillard reaction.

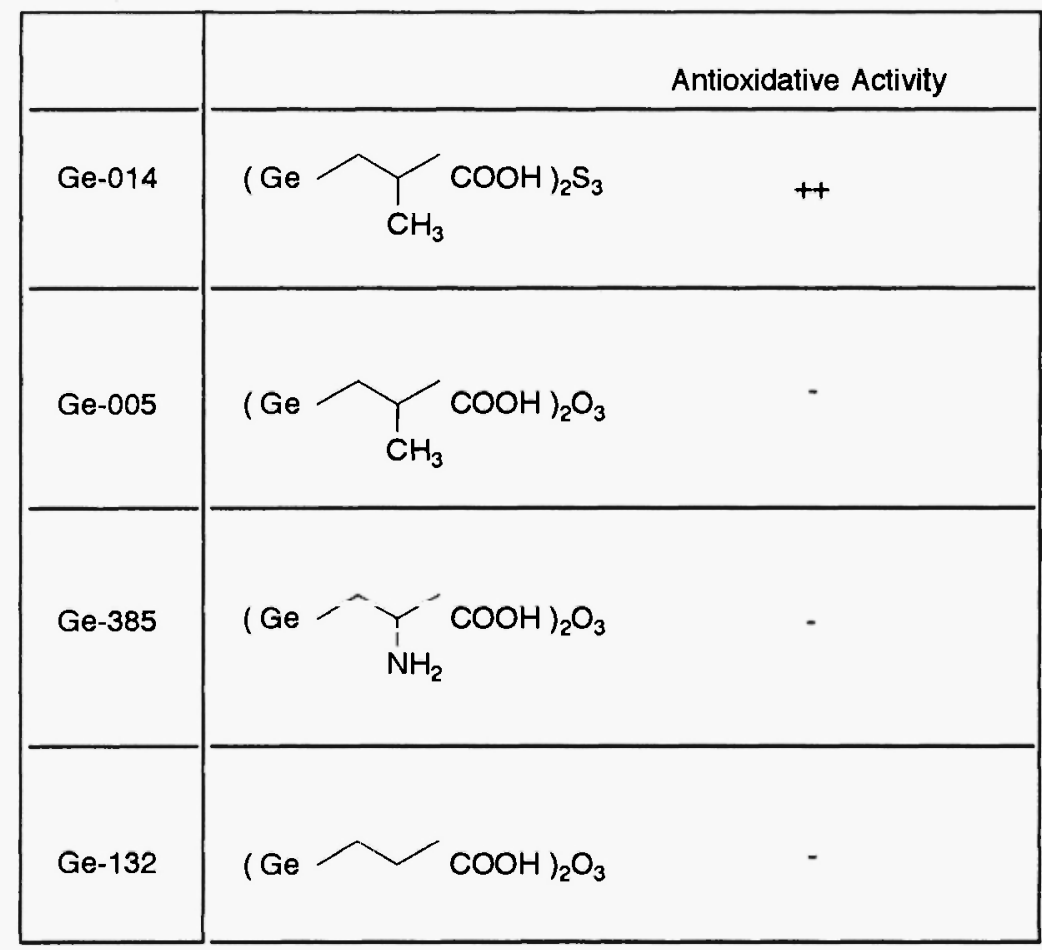

Table I Strctures and antioxidative activity of organogermanium compounds 


\section{Interaction of organogermanium compounds with glucose}

From these backgrounds, we speculated that interraction between Ge-132 and glucose must play an important role for inhibitory effects on the biological Maillard reaction. As shown in Fig. 4, it is possible that glucose may have five different forms, however, glucopyranose strctures of glucose are supposed to be dominant in the hydrophilic solutions and glucose in aldehyde form present between two anomers is speculated to be the most important form for the progress of the Maillard reaction because of its reactivity. In the $1 \mathrm{H}$-NMR spectrum of glucose in D2O, $\beta$-oriented $\mathrm{C}-1$ proton of $\alpha$-anomer of glucopyranose is observed at $\delta 5.2$ and $\alpha$-oriented $\mathrm{C}$ - 1 proton of $\beta$ anomer of glucopyranose is observed at $\delta 4.6$ ( $\alpha$-anomer: $\beta$-anomer $=37: 63$ ). On the other hand, Ge-132 is dissolved in water upto $0.98 \%$, and also dissociated to monomer, trihydroxygermylpropanoic acid. In $1 \mathrm{H}$-NMR spectrum of the 1:1 mixture of glucose and Ge-132 (Fig. 5), newly formed doublet was observed at $\delta 5.68$, which was speculated to be $\mathrm{C}-1$ proton of glucose interacting with Ge-132, however, it is not clear whether this complex has glucopyranose or glucofuranose structures. In order to confirm the structure of glucose:Ge-132 complex, some furanose type derivatives which have the protection groups at the site of 3- and 5-position to fix 5member ring structure. In $1 \mathrm{H}$-NMR spectrum of the 1:1 mixture of d-glucofuranurono-6,3-lactone and Ge-132 in D2O, newly formed doublet at $\delta 5.75$ is ascribed to proton at C-1 position of furanose-type ring structure. The same proton peak is also observed in the case of 3,5-acetone glucose which has the same fixed furanose-type ring structure, however, no peak was observed in the case of cyclohexane-1,2-diol (Fig. 6). From these data, it is concluded that Ge-132 can make a complex only with glucofuranose structure.

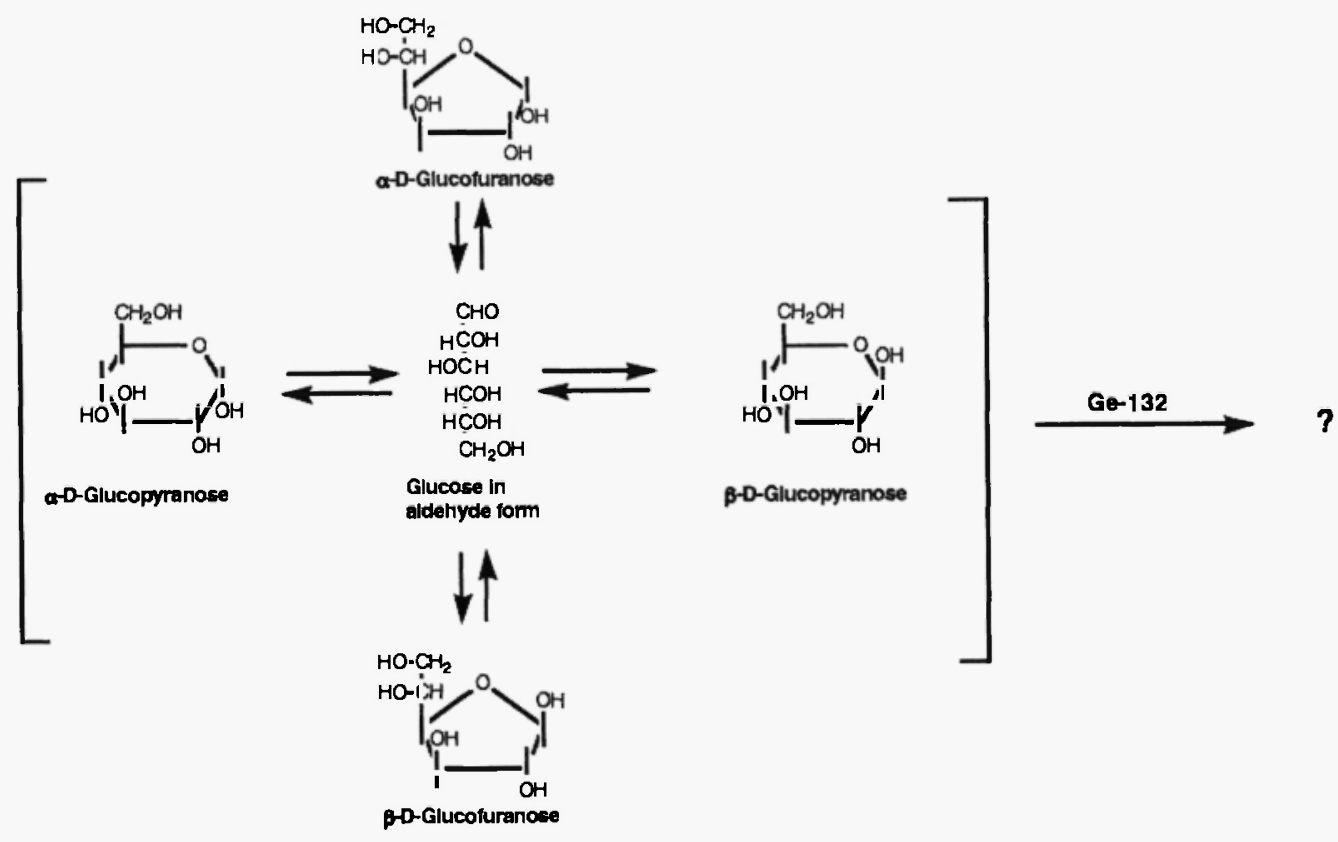

Fig.4 Mutarotation of glucose in the aqueous solution 


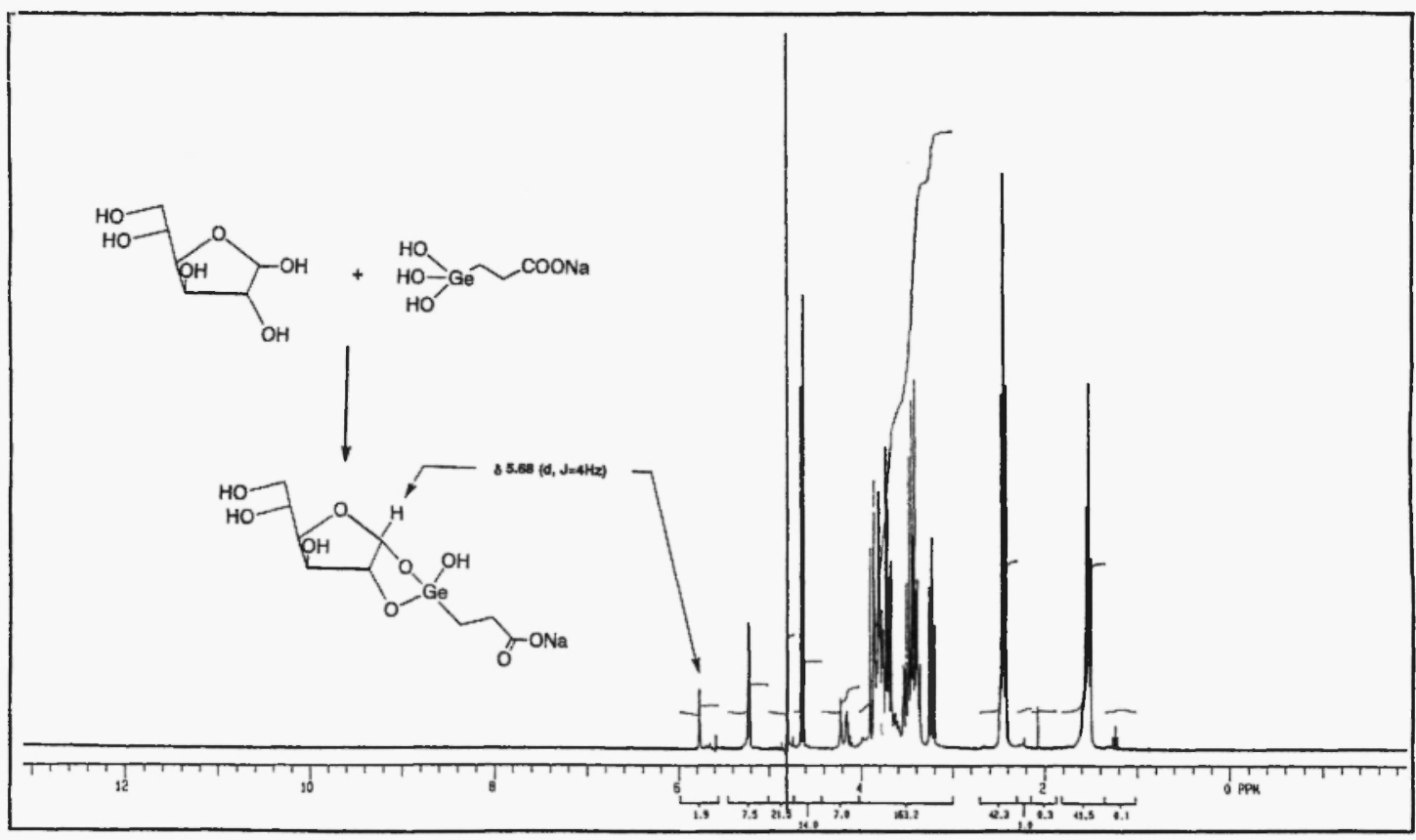

Fig.5 ${ }^{1} \mathrm{H}$-NMR spectrum of 1:1 mixture of Ge-132 and glucose in D2O

Interaction with Ge-132

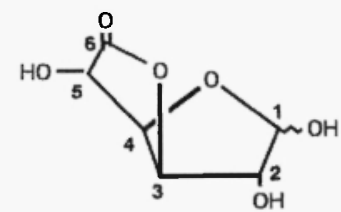

D-Glucuronolaclone

(D-Glucofuranurono-6,3-lactone)

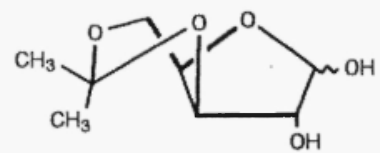

3,5-Acetoneglucose

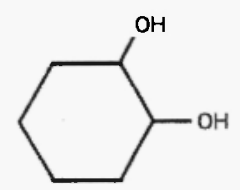

Cyclohexane-1,2-diol
$+$

$+$

Fig.6 Interaction of furanose-type derivatives with Ge-132 


\section{DISCUSSION}

The formation of Advanced Glycation End Products (AGE) by the Maillard reaction may possibly be the major factor of many diseases such as diabetic complications, mutation and finally aging 12). Maillard reaction is the reaction between amino group of proteins and sugars, in particular glucose. Glucose and amino-group of proteins form Schiff bases, whose reaction is reversible, and then Amadori products can be formed by rearrangement from the Schiff bases. As shown in the previous paper ${ }^{3)}$, glucose was reacted with $\mathrm{N}^{\alpha}$-tert-Boc-Lysine at $\mathrm{pH} 7.4$ in vitro to form a Schiff base adduct which undergoes an Amadori rearrangement to $F L$, and effects of organogermanium compounds on formation of FL was examined by HPLC. These data showed that organo-germanium compounds, in particular Ge-132 inhibited dose-dependently and completely the formation of FL and also found to inhibit the further progress reaction of FL to brown pigments.

Many different types of AGE can be formed from Amadori products in the progress of the biological Maillard reaction. Recent many reports indicated that the formation of pentosidine must be the important biological and physiological marker of the Maillard reaction 10 ). Recently, Baynes reported that there are significant difference of the level of fuructoselysine and pentosidine between diabetic and non-diabetic patients. By our investigation of inhibitory effect of organogermanium compounds on the formation of pentosidine, it was found that organogermanium compounds, in particular Ge-132 inhibited the formation of pentosidine although the detailed mechanism was not clear. Ge-132 was also found to prevent the formation of AGE from Amadori products to a certain extent, and these data indicated that Ge-132 inhibited effectively the reaction of glucose and amino acid, and also the formation of AGE from Amadori products.

By the detailed ${ }^{1} \mathrm{H}$-NMR analyses of the reaction of $\mathrm{Ge}-132$ with glucose or chemically synthesized glucofuranose derivatives, it was found that Ge-132 made an complex with glucofuranose form of glucose and inhibited the early stage of the Maillard reaction. At the present stage, it is not clear whether Amadori compound has furanose structure or pyranose structure, however, it is proposed that Ge-132 can make a complex with furanose ring structure of Amadori compound as shown in Fig. 7, because Ge-132 was found to prevent the progress of Maillard reaction at the late stage from Amadori compound. Now, chemical analyses whether other organogermanium compounds can interact with furanose structure of glucose are in progress.

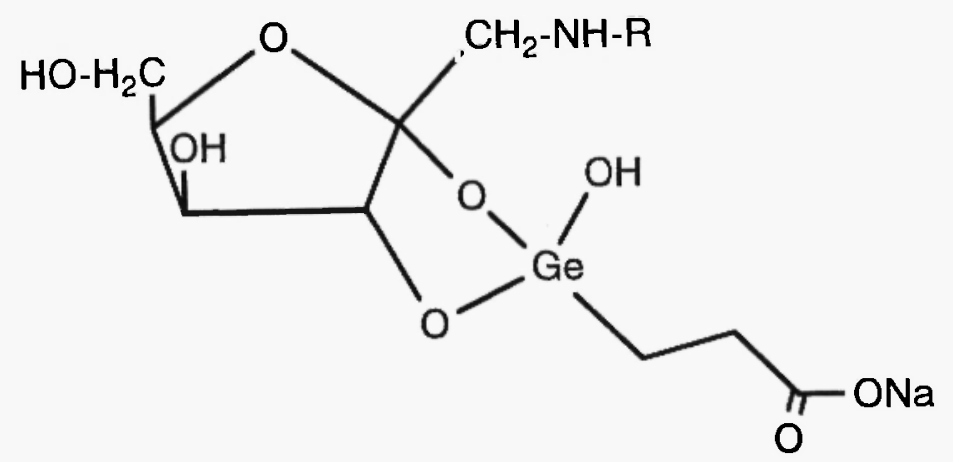

Fig.7 Proposed structure of Ge-132--Amadori compound complex 


\section{ACKNOWLEDGMENT} discussion.

The authors wish their thanks to Professor K. Nakamura, Kitasato University for stimulating

\section{REFERENCES}

1) S.A.Kaars, F.R.Rijkens, G.J.M. Kerk and A.Mante, Nature, 201, 736 (1962)

2) K.Asai and N.Kakimoto, Chem. Abs., 80, 112665d (1974)

3) N.Kakimoto, K.Miyao and M.Akiba, in "Frontiers of Organogermanium, Tin and Lead Chemistry" (E.Lukevics and L.Ignatovich, eds.), p.319, Latvian Institute of Organic Synthesis, Latvia (1993)

4) L.C.Maillard, C.R. Acad. Sci., 154, 66 (1912)

5) M.A.Brownlee and H.Vlassara, N. Engl. J. Med., 318, 1315 (1988)

6) K.Nakamura, K.Nomoto, K.Kariya, Y.Nakajima, H.Nishimoto, S.Uga, M.Miyata, T.Osawa, S.Kawakishi and N.Kakimoto, Amino Acids, 1, 263 (1991).

7) N.Kakimoto, M.Akiba and T.Tanaka, Synthesis, 272 (1985)

8) N.Kakimoto, M.Matsui, T.Takada and M.Akiba, Heterocycles, 23, 2681 (1985)

9) F.G.Njoroge, A.A.Fernandes and V.M.Monnier, J. Biol. Chem., 263, 10646 (1988)

10) D.R.Sell and V.M.Monnier, J. Biol. Chem., 264, 21597 (1989)

11) T.Osawa, A.ide, J.D.- Su and M.Namiki, J. Agric. Food Chem., 35, 808 (1987)

\section{Received: September 24, 1993}

